\title{
Réflexions sur le sacrifice dans la religion traditionnelle des Bamoun (Cameroun)
}

Thoughts about sacrifice in the traditional religion of the Bamum (Cameroon)

\section{Claude Tardits}

\section{(2) OpenEdition}

\section{Journals}

Édition électronique

URL : http://journals.openedition.org/span/435

DOI : 10.4000/span.435

ISSN : 2268-1558

Éditeur

École pratique des hautes études. Sciences humaines

\section{Édition imprimée}

Date de publication : 1 septembre 1979

Pagination : 117-129

ISSN : 0294-7080

\section{Référence électronique}

Claude Tardits, "Réflexions sur le sacrifice dans la religion traditionnelle des Bamoun (Cameroun)",

Systèmes de pensée en Afrique noire [En ligne], 4 | 1979, mis en ligne le 04 juin 2013, consulté le 30 avril 2019. URL : http://journals.openedition.org/span/435 ; DOI : 10.4000/span.435 
R E F L E X I O N S S U R L E S A C R I F I C E

$D A N S$ L A R E I I G I O N T R A D I T I O N N E L L E

$D E S \quad B A M O U N \quad(C A M E R O U N)$

par Glande Tardits

Le sacrifice était, dans la reijgion traditionnelle bamoun, un acte rituel réservé : à la différence de la prière, pratiquée par tous, il n'était accompli que par le roi et les chefs de lignage. I etait associé ainsi à l'exercice du pouvoir politique ; il apparât en quelque sorte comme un acte de pouvoir.

Cette situation contribue à rendre difficile son étude. Dans cette société en cours d'islamisation et de christianisation depuis le début du siècle, la population êprouve quelques hésitations, parfois quelques rêpugnances, à évoquer la pratique et les croyances prëislamiques; à cette prêvention vient alors s'ajouter le fait que le nombre de ceux qui ont accompli des sacrifices a largement diminue avec le cemps, alors qu'ils ne représentaient déjà qu'une mince couche de la population. Le dernier souverain traditionne1, Njoya, est lui mort en 1933.

Notre rêflexion part donc de notes qui reconstituent le passé bieri plus qu'elles ne reflètent le présent; elles se sont toutefois trouvées enrichies par le parti que l'on a pu tirer de la tentative faite par le roi Njoya d'elaborer, en 1916, un islam bamoun. Ce dernier porte en effet la marque de la tradition préislamique, aidant ainsi indirectement à la saisir.

Dans la première partie de cette conférence, nous dirons quelques mots de l'organisation de la société, ce qui permettra de saisir le contexte dans lequel prenaient place les activités religieuses. Nous esquisserons d'abord les traits généraux de la société pour jeter ensuite un coup d'oeil sur l'organisation lignagère, et finalement, rapprocher ces deux niveaux successivement abordés. 
Au début du siècle, le royaume bamoun comptait quelque 70000 habitants qui occupaient, entre le Noun et le Mbam, un plateau de 8000 $\mathrm{km}^{2}$. La sociêté était fortement stratifiēe. A la base de la pyramide par laquelle on aurait pu la reprēsenter, une masse servile englobait près des deux-tiers de la population ; cette masse était formée par la descendance des captifs provenant des guerres du XIXe siècle ou par des prisonniers récents rëduits en esclavege. I1 y avait là une population essentiellement aliénable, propriêté des agnats des lignages dont nous allons parler et du roi. Elle pouvait se trouver grossie d'éléments réduits en servitude à titre de sanction. Les esclaves étaient, il faut le souligner, toujours rattachés à un lignage ou au monarque.

Le tiers restant de la population formait une noblesse dont les membres étaient rêpartis entre quelque 700 patrilignages, noblesse inaliénable, dont le statut n'était toutefois pas opposable au roi. Une trahison politique entraînait la servitude.

Comment s'était constituée cette noblesse? Du fait de I'installation, au fil du temps, des fils et des grands serviteurs des dix-sept souverains qui, depuis la fondation du royaume, vraisemblablement au XVIe siècle, s'étaient succédẻs au pouvoir. Chaque patrilignage remontait à un fondateur nommé, et à un règne qui en indiquait donc l'ancienneté.

Le processus par lequel s'était développée la société était resté le même pendant les trois siècles séparant la fondation de 1'Etat bamoun du démantèlement de ses institutions politiques : il avait abouti à la constitution, d'une part, d'une noblesse princière représentée par les groupes de descendance issus des princes, d'autre part, d'une noblesse palatine formée par les decendants des grands serviteurs. Les guerres victorieuses du XIXe siècle avaient enrichi cette noblesse : les terres conquises avaient êté peuplêes et mises en exploitation par les captifs dont nous avons parlé et les domaines découpẻs sur ces terres attribués par le roi à une partie des chefs de lignage ou gardés par lui.

La sociêtê bamoun avait toutes les apparences d'un immense lignage maximal formë de subdivisions, les lignages princiers ou palatins, qui tous, à travers leur fondateur, s'articulaient sur la lignée des rois. Cette dernière jouait le rôle d'axe générateur dans le royaume. Les esclaves, propriété des membres des lignages et du monarque, n'étaient que des prolongements des groupes de descendance. 
Prêcisons toutefois que la société n'était pas totalement coextensive à cet immense lignage maximal car, sur le territoire du pays, quelques populations battues avaient êté laissees en place, dont les chefs s'etaient soumis au monarque bamoun. On n'abordera néanmoins pas le problème de leurs rapports avec 1'autorité centrale.

Venons-en au deuxième point de cette première partie : quels caractères présentaient les lignages bamoun ?

Indiquons, en première observation, que le lignage avait un double aspect. Il constituait une unité parentale puisqu'il était formé par la descendance agnatique du fondateur, a laquelle s'ajoutaient parfois les enfants des filles non mariêes, qui étajent donc restées liêes à leur groupe d'origine. Cette unite avait une extension et une profondeur variables: les groupes les plus anciens pouvaient s'étendre sur quinze à vingt niveaux généalogiques. Il formait ensuite une unite politique : il fournissait à l'Etat sa seule organisation territoriale et le chef de lignage représentait le roi, dont il était le relai, sur la terre occupée par les siens.

Deuxième observation : les patrilignages restaient toujours localisês sur un territoire d'un seul tenant. Les localisations dépendaient initialement du statut du fondateur du groupe : les grands serviteurs étaient installés à faible distance de la résidence royale, alors qu'on établissait les princes au-delà de cette ceinture, ce qui entraînait le rejet regulier vers l'extérieur des lignages issus des fils des rois précédents. Ces mouvements n'entrâñaient néanmoins aucune dispersion des nembres du groupe. Au XIXe siècte, lorsque la ville de Foumban se constitua, les citadins finirent toutefois par manquer de terre, et il arriva que des familles de la noblesse émigrent vers les domaines décou-pés dans les terres conquises, mais ces emigrés conservaient leur maison dans le groupe d'origine, te demeuraient soumis a son chef. Le Ijgnage conservait done son unité.

Troisième point : le lignage était une entité juridique. La collectivite détenait les droits fonciers sur les terres occupées par ses membres, qu'il s'agisse de l'espace d'installation du groupe on des domaines attribués par le roi. Elle formait une unité judiciaire et religieuse: on réglait les diffêrends en son sein, à moins qu'ils n'aient relevë, en raison de leur gravite, de la justice du roi. La prospérité du groupe dépendait de la ligrêe d'ancêtres représentés par les chefs de lignage 
décédés depuis sa fondation. Enfin, dans les guerres, les membres de la collectivité combattaient ensemble.

Quatrième trait, le lignage était fortement différencié. A l'intérieur du groupe, chacun établissait sa généalogie en remontant jusqu'au chef de lignage dont sa ligne d'ascendants était issue. Cette ligne des chefs à laquelle tous se rattachaient êtait 1'axe gênérateur du lignage. Les agnats relevant du même niveau généalogique, quel que soit leur degré de parenté, entretenaient des relations fraternelles ; ceux qui relevaient de niveaux consécutifs se traitaient comme des pères ou des fils. Au sommet du lignage, le chef du groupe occupait, en raison de la règle de succession universelle, une position de père vis-à-vis de tous les membres de la collectivité. Chaque nouveau chef succédait en effet à la personne de son prédécesseur, et se trouvait ainsi mis au point de départ de toutes les lignées d'agnats issues des fils des chefs successifs.

I1 allouait les ressources du groupe; attribuant, et retirant éventuellement, les droits sur les terres ; il conduisait ses parents à la guerre, et répartissait ensuite le butin, quand il y en avait, que lui remettait le roi pour sanctionner les exploits des siens. Il réglait les différends qui surgissaient au sein du lignage : il devait détecter les sources des malheurs qui le frappaient et en éliminer les causes. I1 officiait enfin comme prêtre des ancêtres.

Dernier point : 1 'unitê du lignage était toujours préservée. Aucune fission n'était permise, et aucun lignage bamoun ne s'est formé après que des agnats se soient séparếs d'un lignage déjà constitué. Les patrilignages ne se développaient qu'à partir de l'établissement d'un fils ou d'un grand serviteur du souverain; et tout autre lien entre le grand serviteur et ses ascendants se trouvait alors coupé. Les migrations vers la campagne permettaient de réduire les tensions entre agnats sans détruire 1 'unitê du lignage. Lorsque les conflits intërieurs prenaient de 1 'importance, les éléments qui en étaient tenus pour responsables, se serait-il agi du chef de lignage lui-même, étaient remis au roi et réduits en servitude. Il y a plus, les conseillers du monarque et les grands officiers du palais se recrutaient tous parmi les chefs de lignage, de sorte qu'il ne s'était institué dans le royaume aucune hiérarchie distincte, par son origine, de celle des chefs de lignage.

Nous terminerons ce rapide examen de l'organisation de la sociêtë 
en rapprochant les pouvoirs du chef de lignage de ceux du roi.

Précisons, pour commencer, que les liens entre les chefs de lignage et le roi étaient similaires à ceux qui avaient lié, lors de la fondation du groupe, le monarque au fondateur. Cela en raison de la règle de succession universelle qui s'appliquait à toute la société : lorsqu'un lignage avait été fondé par un prince, un lien agnatique était donc perpétué ; lorsqu'il I'avait été par un grand serviteur, c'est une relation plus variée qui se trouvait préservëe, un grand serviteur pouvant entretenir avec le monarque une relation utérine, un rapport mystique ou même un simple lien de subordination qui se trouvait souvent transformé en rapport de consanguinité utérine. Nous laisserons toutefois de côté ici l'analyse de ces situations qui ne concernent pas directement notre propos. Quels qu'ils aient été, ces liens établissaient entre le monarque et le chef de lignage des rapports de parenté réels ou fictifs.

Deuxième notation, le développement génétique de la société, qui était sous-jacent à son développement historique, s'était accompagné du maintien d'une relation d'homologie entre 1 'organisation des pouvoirs au niveau des chefs de lignage et à celui du roi. Le souverain allouait les richesses à l'échelle du pays, attribuant et retirant aussi bien les terres lignagères que domaniales ; il prenait la tête de l'armée bamoun lorsqu'elle partait en guerre et répartissait êventuellement au retour le butin ; il détenait en matière de justice le droit de vie et de mort ; enfin, il était le prêtre des souverains décédés et ses activités religieuses affectaient la vie de tout le pays.

Remarquons, pour terminer avec ce rapprochement, que les différences d'échelle auxquelles s'exerçaient les pouvoirs respectifs du chef de lignage et du roi leur confêraient quelques traits distinctifs : c'est ainsi que le roi qui disposait d'un volume considërable de ressources, pouvait agir sur la stratification même de la société, et entretenir une opposition entre la noblesse de sang et la noblesse palatine ; l'importance du tribut perçu annuellement lui permettait encore d'agir comme régulateur de l'économie en temps de disette. Le droit de vie et de mort était le privilège exclusif de la souveraineté. Le culte des ancêtres royaux avait des trais particuliers ; $i 1$ comportait un culte aux crânes qu'on ne retrouvait pas dans les 1 ignages.

Nous devons retenir, pour notre propos, la similitude entre les rôles religieux des chefs de lignage et du roi : ils étaient en effet les seuls qui aient eu le droit d'exécuter des sacrifices, car c'est 
entre leurs mains qu'ëtait concentrểe l'autorité.

Cette brève esquisse permet de comprendre les raisons pour lesquelles nous avons pu écrire que le sacrifice avait toutes les apparences d'un acte de pouvoir.

Passons maintenant à la seconde partie de cette conférence et examinons la pratique sacrificielle. On la situera d'abord dans l'ensemble des recours religieux ; on examinera ensuite la place qu'elle garda dans I'islam bamoun diffusé pendant les années 1916 et 1917. Ceci nous permettra de formuler les quelques réflexions que suggère l'examen des matêriaux dont nous avons disposé.

Les recours religieux sont de deux ordres : ceux qui sont ouverts à tous, la prière et les libations, et ceux qui sont réservés aux détenteurs de 1'autorité, les sacrifices. Ils forment néanmoins un ensemble, la fonction et la signification des uns éclairant celles des autres.

Le nzuom, terme par lequel les Bamoun désignent les prières, est non seulement ouvert à tous mais il se pratique à tout moment et en tous lieux. C'est un recours universel susceptible d'être utilisê en un nombre indéfini de circonstances.

Voyons quelques ênoncés de nzuom :

1. "Si je lance un caillou vers une feme mariêe, si je la regarde deux fois, que celui de jour ou celui de nuit ne me lâche pas". Clarifions la formule : "que le malheur m'atteigne si je tente de me faire remarquer d'une feme mariée".

2. "Si j'ai volé le vin du champ de raphia d'autrui pour le boire, que celui de jour ou celui de nuit me saisisse et ne me lâche pas".

3. "Si je prêtends ne rien savoir alors que je sais quelque chose, que celui de jour ou celui de nuit me saisisse et ne me lâche pas".

La construction de ces énoncés est toujours la même : une proposition subordonnée conditionnelle se référant à une transgression, et une principale appelant une sanction si la condition se trouve réalisée.

Nous avons affaire à des formules imprēcatoires qui ont des objectifs divers. Elles peuvent servir à prévenir un malheur, lorsque l'orant se sent menacé par un mauvais présage, par exemple à la suite d'un mauvais rêve; elles peuvent permettre à une personne accusée, à tort à ses yeux, de se disculper ; enfin un náuom peut être utilisé pour écarter un malheur qui a commencé de vous atteindre, pour mettre, par exemple, un terme à une maladie.

Dans un univers où échec, querelle, disette, maladie, mort renvoient 
à des transgressions morales, I'universaititè du recours au nduom s'explique. On comprend donc la grande flexibilité des formules que I'on peut utiliser puisque $l$ 'orant les adapte aux circonstances. Le natuom n'a pas, de plus, de destinataire précis. Les Bamoun interrogés à ce sujet sont souvent embarrassés. Ils feraient appel aussi bien aux ancêtres qu'à ces divinitës ubiquistes que les Bamoun dêsignent sous le nom de panyimyi. Cette imprëcision, notons-1e, convient à une sociêté où une large fraction de la population vit loin des tombes de ses ancêtres, dont elle a été séparee par les guerres.

Une question importance pour l'interprétation de la prière come de 1 'ensemble des pratiques rituelles se pose: Ie nzuom est-il toujours tenu pour efficace? Ecarte-t-il toujours les malheurs ? La réponse tient dans 1 'usage que $l^{\prime \prime}$ on en fait et, en particulier, dans le fait qu'il sert aux accusês à établir leur innocence. Personne, d'après les témoins, ne s'engageait par un nzum s'il se savait coupable. Aujourd'hui encore, peu de gens sont susceptibles de défier les vieilles croyances.

Les libations eraient fréquentes : elles se faisaient en dehors des sacrifices, bien qu'elles les aient toujours accompagnés. Pratiquées par tous, elles se faisaient néanmoins en des lieux particuliers et étaient obligatoires en certalnes circonstances. On utilisait des boissons fermentées, biëres de sorghio, de maïs ou vin de palmier raphia, considérées conme des vêhicules de force. Les liquides ëtaient versés au foyer d'une famille, sur une combe, dans des trous à libations creusés dans les lieux de rëunion des sociétés secrètes; on pouvait aussi faire des offrandes à la terre en versant quelques gouttes sur le sol, là où l'on se trouvait. Ces oftrandes se faisaient aussi bien à l'occasion d'un repas, de la réunion des membres d'une société que lors des grandes fêtes annueiles où elles accompagnaient alors les sacrifices. Destinées à la terre, aux ancêtres, aux panyinyi, les libations servaient aussi bien à apaiser qu'à remercier et propicier.

Le sacrifice diffère des praciques précédentes, nous 1 'avons dit, en raison de son caractëre réservé et des précautions qui sont prises pour le iui conserver. Il est exécuté pax les chefs de lignage et le roi aux grandes fêtes du calendrier agricole et religieux traditionnel. On sacrifiait en juillet, après la récolte de maís qui était suivie de la remise du tribut au palais. Au debut du siècle, le tribut représentait des dizaines de tomes de denrês: maǐs, sorgho, huile et viande. 
Une fête générale, dite fête des nguón, intervenait à ce moment ; les nguơn êtaient des tambours à friction que leurs possesseurs amenaient à cette occasion au palais et qui, en principe, ne devaient être vus ni par les femmes, ni par les enfants. Lors de cette manifestation, le roi devait écouter les doléances recueillies dans la population et prendre des mesures destinêes à éliminer les abus et même les violences qui provoquaient les maux dont souffrait le pays, disettes, troubles, épidemies. Une immense agape avait lieu à la résidence du roi et la manifestation se terminait par des sacrifices exécutés dans les cimetières de tous les chefs de lignage, sur les tombes de leurs ancêtres, dans celui du roi et dans le sanctuaire où l'on conservait les crânes royaux.

On cêlébrait en dêcembre, à la saison sèche, une autre fête dite de $n \succsim a$. Elle donnait lieu à une grande manifestation chorégraphique, et offrait à chacun 1'occasion de faire étalage de ses plus beaux atours. C'était, disent les Bamoun, la "fête de la beaute". On exêcutait encore des sacrifices dans tous les cimetières du pays et au sanctuaire royal comme pour le nguón.

En dehors de ces fêtes fixes, on sacrifiait encore, avant les départs en guerre, après les grandes chutes de pluie marquées par l'apparition d'un arc-en-ciel, et à chaque fois que la divination le prescrivait.

Les chefs de lignage ou le roi assistês respectivement de leur intendant ou des grands officiers du palais procédaient à 1'exécution du sacrifice. Les intendants étaient d'origine servile et les titamfon appartenaient à la noblesse palatine, ce qui leur permettait de mettre la main à l'exécution des sacrifices. On égorgeait des volailles, des chèvres, des moutons et des bovins. Avant de tuer l'animal, le sacrificateur faisait une libation sur les pierres tombales des cimetières ; dans celui du roi, on versait le liquide dans les défenses d'ivoire qui émergeaient de chaque tombe. Les monolithes recevaient ensuite le sang sacrificiel. La corne à libation et les couteaux sacrificiels utilisés dans le rituel étaient soigneusement cachës après avoir servi. Libations ot sacrifices s'accompagnaient de l'énoncé d'un nzưm.

$D^{\prime}$ après quelques témoins, la réaction du liquide qui pouvait ou non jaillir en moussant hors des défenses, au cimetière royal, ou la position de 1 'animal sacrifié indiquaient si le sacrifice avait été ou non agréé. Les sacrifices paraissent avoir une double finalité : exécutés à $1 a$ 
suite de quelque maiheur, avant les départs en guerre et même à la fête des.nguón, on leur demandait de mettre un terme à 1 'infortune ou de l'empêcher. Après les grandes chutes de pluie et à la fête de nža, ils semblent bien destinés à remercier les ancêtres et les dieux (pqnyinyi).

L'accès aux lieux sacrês et leur utilisation étaient rendus difficiles à tous ceux qui en étaient écartés par la tradition. Tout agnat, frère ou fils du chef de lignage ou du roi, qui aurait tenté de s'y rendre êtait réduit en servitude: De plus, le matériel sacrificiel était dissimulé. Celui qui tentait de violer la règle êtait accusé d'usurpation, et l'on redoutait que le contrevenant ne se serve du pouvoir qu'il aurait tenu de son rapport avec les ancêtres.

Soulignons, pour terminer, deux traits tout-à-fait caractéristiques des pratiques que 1 'on vient d'évoquer. Ces pratiques ont une fonction évidente : elles servent à agir directement sur les rapports sociaux. On utilise la prière pour se disculper et l'acte religieux constitue un vëritable mode de preuve, qui pernet de détecter les causes des malheurs ; quant aux libations et aux sacrifices, ils servent a apaiser et éventuellement à mettre un terme aux afflictions. Secondement, si le sacrifice est bien un acte fort, apparemment contraignant -- car comment comprendre autrement son caractère réservé et protégê --, la prière est. aussi un acte dont l'efficacité est tenue pour acquise.

Que nous apprend 1 'examen du projet religieux que Njoya élabore en 1916 ? Rappelons d'abord les faits : en décembre 1915, les Allemands et la Mission protestante de Bâle, présents dans le pays depuis le début du siècle, quittaient le royaume. Les troupes anglaises s'instaliaient à Foumban. Le départ de la Mission modifia alors la situation religieuse compliquée, qui se développait dans le royaume depuis près de vingt ans. En effet, entre 1896 et 1898, était intervenue une manière de conversion à l'islam. Celle-ci se produisit à la suite d'une intervention des Peuls de Banyo sollicités par le jeune Njoya, qui venait de monter sur le trône, et se trouvait aux prises avec une guerre civile. La "victoire du cheval" conduisit le roi à demander aux Peuls islanisés l'envii de marabouts. Elle l'incita encore à élaborer une écriture, ce qui fut fait quelques temps plus tard. Les marabouts hausa introduisirent à la cour royale la pratique des prières musulmanes et même celle du jê̂ue. Les Bamoun n'abandonneirent pas pour autant leurs propres rites. Dans l'esprit du roi, les prières musulmanes s'ajoutaient aux recours religieux 
traditionnels, et leur usage devait conférer aux orants la puissance dont avait fait montre la cavalerie peule. Le rite apparaît, ici encore, comme un instrument du pouvoir.

Quelques années plus tard, les Allemands parvenaient à Foumban. En 1906, Njoya séduit par l'oeuvre scolaire qui accompagnait la présence des Missions européennes autorisait la Mission de Bâle à établir un poste dans sa capitale. Les rapports avec les nouveaux venus furent excellents. Non seulement le monarque favorisa la scolarisation du pays, mais il suivit les prédications et assista même aux services religieux. Des fragments de la Bible furent traduits en bamoun, en utilisant l'écriture mise au point par le roi. L'action évangélisatrice eut toutefois une limite : les missionnaires ne purent convertir Njoya, qui possëdait plusieurs centaines d'épouses qu'il n'était pas question de renvoyer. Les rapports entre la cour et les protestants n'en restèrent pas moins cordiaux. Il va de soi que les pratiques traditionnelles ne furent pas plus abandonnées pendant les dix ans de présence de la Mission qu'elles ne 1 'avaient été à la fin du XIXe siècle, après la première diffusion de 1 'islam.

Les années de relations entre Njoya et les représentants de l'islam et du christianisme, marabouts et pasteurs, avaient familiarisé le monarque avec les doctrines de salut. En 1916, les marabouts n'avaient pas encore retrouvé leur influence et les chrëtiens étaient partis. Le roi profita de cette circonstance pour élaborer les préceptes d'une religion en rédigeant un petit ouvrage dont le titre est en français "Poursuis pour atteindre". Il y avait là, ramassés dans une trentaine de chapitres, les éléments de ce que 1 'on pourrait appeler une religion nationale inspirée par l'islam. Plusieurs passages reprennent, sans modifications d'ailleurs, le texte de la Risâza.

Njoya fit à l'époque reconstruire une mosquée où l'on enseignait la doctrine royale. L'entreprise dura peu : elle s'étendit certainement sur une partie ou sur la totaljté de l'année 1916. Les archives rédigêes par les Français qui avaient, à l'époque, remplacé les Anglais, en font foi. Elle s'est peut-être prolongêe quelques temps sur 1'année 1917. Le monarque était en effet soumis aux pressions des marabouts toujours présents dans le pays, qui l'accusaient de jouer au prophète, et à celles de quelques-uns de ses fils dẹjà convertis. Aussi Njoya finit-il par renoncer à son projet pour revenir à l'orthodoxie musulmane. Lorsqu'il 
Le fit, ce fut pour s'engager dans une politique de conversion du pays. La conversion prit de $I^{\prime}$ ampleur dans les années 20 , et 1 'islamisation se fit en partie en rêaction à la colonisation, qui s'était engagée dans une lutte contre le roi et s'appuyait localement sur d'anciens élèves de La Mission protestante.

Quel est le contenu de la doctrine royale? Les principes théclogiques en sont les suivants : i) I'unicité de Dieu est affirmèe : Dieu est inengendré, omniscient, ubiquiste et ì a crêé le monde auquel il a donné ses lois;

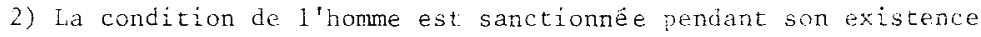
terrestre, et il peut être puni par les malheurs et les fléaux envoyés par Dieu ; elle est encore sanctionnée après sa mort : le monde connaît une fin, puis une résurrection intervient, à la suite de quoi l'home est voué, toujours en fonction de sa conduite, à une éternité de soufErances dans le feu ou à une felicité sans Ein.

3) Les préceptes à respecter pour éviter les malheurs sur cette terre et pour jouir du bonheur éternel sont les lois de la société, voulues, nous l'avons dit, par Dieu - les règles enumétés par Njoya sont tout simplement celles de la morale traditionnelle bamoun -- ; 1'home doit encore pratiquer ce que le monarque appelle les "actes aimes de Dieu", c'est-à-dire les rites dont la descrintion remplit les deux-tiers de son ouvrage. Notons finalement que le Seigneur peut être miserricordieux envers celui qui se repent.

Quels sont" les "actes aimés de Dieu" ? Les prières, que l'on doit accomplir cing fois par jour, celle du vendredi se faisant collectivement à la mosquee, les jeûnes qui correspondent à ceux requis dans l'islam, le sacrifice du mouton aue l'on fait le 10 e jour du dernier mois de l'amnée et enfin l'aumône.

On est manifestement devant une pratique directement reprise de l'islam; le texte royal demenre toutefois somnaire. Rien, par exemple, n'est dit de la puritication rituelle qui, chez les musulmans, est fondamentale pour la validation des actes religieux ; l'obligation de jeuner est sommairement indiquêe, sans que soient envisagêes les circonstances dans lesquelies ies croyants peuvent se trouver engagés. Il est possible que le roi ait pensé, pour pallier les lacunes de son texte, a utiliser celui de la Risẩa. La chose est simplement vraisemblable. 
Si l'on s'en tient aux obligations essentielles de l'islam, à ses piliers, les traits distinctifs de la doctrine de Njoya sont évidents : le roi n'en a retenu que trois, la prière, le jeûne et l'aumône ; il en a omis deux, la profession de foi dont 1'énoncé n'est, dans son texte, prévu que lorsqu'on embrasse la nouvelle foi, et le pélerinage. Trait singulier et remarquable, il a conféré au sacrifice un statut qui $n^{\prime e s t}$ pas celui de 1 'orthoḍoxie. Il en a fait un "acte aimé de Dieu" alors que, dans le rite mâlikite, le sacrifice de 1'Aïd-e1-Kebir est d'ordre traditionne1 et non divin. C'est un acte de rachat, d'expiation qui est simplement recommandé (Chelhod : 55-56). Ajoutons que la langue du culte imposée par Njoya est le bamoun. On est, devant cette entreprise, toutà-fait fondé à y voir une tentative du roi pour donner à son peuple une religion nationale, qui prend 1 'aspect d'un islam bamoun.

Dans ce texte et ce projet, nous relèverons deux traits : I'un vient compléter notre propos sur le sacrifice, 1'autre nous introduit aux problèmes que ne peut manquer de poser la diffusion de 1'islam.

La tradition préislamique nous met en présence d'une pratique religieuse où $I^{\prime}$ homme agit sur le surnaturel, qu'il semble bien contraindre par la prière et le sacrifice. La portêe de celui-ci est telle qu'on le réserve aux hommes de rang. Dans la société bamoun, la fonction sociale des rituels paraît fondamentale, lorsqu'on les compare à ceux des religions, qui visent avant tout au salut de leur adeptes. Il n'est donc pas surprenant que Njoya maintienne le sacrifice pour en faire un des piliers de sa doctrine. On peut de plus rapprocher de cette position le caractère miséricordieux qu'il attribue à Dieu, alors qu'en regard, la doctrine d'A1 Quayrawânî est, elle, fortement attachêe à la prédestination, et voir en tout ceci la marque d'une pensêe dans laquelle la divinité doit pouvoir être flêchie. L'analyse de la doctrine royale confirmerait ainsi celle qu'on a donnée de la prière et du sacrifice mais, à son tour, cette doctrine ne prendrait son sens que si on la rapproche de la tradition antêrieure. I1 y aurait là une cohérence qui tendrait à valider notre analyse.

Ces observations débouchent sur une interrogation plus génêrale. Comment s'effectue, en fait, dans les sociétés africaines traditionnelles, le passage à une religion qui, d'une part, retire à.la divinité le caractère anthropomorphique que conservent les ancêtres et qui, d'autre part, enlève aux rites leur pouvoir d'engendrer la réciprocité si 
précisément indiquêe dans la fameuse formule do ut des qui fonde l'acte sacrificiel.

Bibliographie.

AL-QUAYRAWAN̂Â, La RisâZa. Alger, 1975.

CHELHOD, J., Le sacmifice chez les Arabes. Recherches sur 1'évolution des rites sacrificiels en Arabie occidentale. Paris, 1955.

NJOYA, Poursuis pour atteindre. Translitéré et traduit du bamoun par

I. Njoya et I. Paré en 1961. Ms 74 du répertoire de I. Dugast et M. D. W. Jeffreys.

TARDITS, C., Le royaume bamoun (sous presse). 
